\title{
mww.casopisimamananpl PAN 级它 \\ of \\ The Effect of Cooling Agent on Stress and Deformation of Charge-loaded Cast Pallets
}

\author{
A. Bajwoluk *, P. Gutowski \\ Mechanical Engineering Faculty, West Pomeranian University of Technology, Szczecin \\ Al. Piastów 19, 70-310 Szczecin, Poland \\ * Corresponding author. E-mail address: Artur.Bajwoluk@zut.edu.pl
}

Received 17.05.2017; accepted in revised form 11.07.2017

\begin{abstract}
The results of research on the effect of the type of cooling agent used during heat treatment and thermal-chemical treatment on the formation of temperature gradient and stress-deformation distribution in cast pallets, which are part of furnace accessories used in this treatment, are disclosed. During operation, pallets are exposed to the effect of the same conditions as the charge they are carrying. Cyclic thermal loads are the main cause of excessive deformations or cracks, which after some time of the cast pallet operation result in its withdrawal due to damage. One of the major causes of this damage are stresses formed under the effect of temperature gradient in the unevenly cooled pallet construction. Studies focused on the analysis of heat flow in a charge-loaded pallet, cooled by various cooling agents characterized by different heat transfer coefficients and temperature. Based on the obtained temperature distribution, the stress distribution and the resulting deformation were examined. The results enabled drawing relevant conclusions about the effect of cooling conditions on stresses formed in the direction of the largest temperature gradient.
\end{abstract}

Keywords: Application of information technology to the foundry industry, Cast grates, Thermal stresses, Heat treatment equipment

\section{Introduction}

During heat treatment and thermal-chemical treatment, furnace components which carry the heat-treated charge are exposed to the effect of the same factors as the heat-treated items. These factors include, among others, high and rapidly changing temperature and strong carburizing atmosphere. Due to the nature of the operation of furnace components, these factors recur in cycles, thus exerting further adverse effect on their lifetime.

Particularly important, and at the same time highly susceptible to the adverse effect of such operating conditions, part of the furnace tooling is the system of cast pallets on which the charge is resting during heat treatment. These castings are open-work thin-walled structures weighing from 8 to $90 \mathrm{~kg}$, typically cast from creep-resistant, high-nickel and chromium-rich austenitic alloys [1-7]. As a result of operation under the conditions described above, as the time elapses, the pallets suffer gradually increasing deformation and the formation of cracks. These damages are the main reason which accounts for the withdrawal of pallets from further use. Since the cost of purchasing new pallets makes a significant part of the operating costs of furnaces, it is important to take proper steps and prolong the durability of these components [4-7], this mainly by learning more about the mechanism responsible for their destruction.

Under the conditions of rapid temperature changes, stresses generated by different thermal expansion of the cast steel components $[5,8,9]$ and by temperature gradient $[5,10,11]$ start forming in the pallet castings. Regardless of the size of the area of the pallet where temperature differences arise, the key problem in the stress development caused by the temperature gradient is the dynamics of the heating and cooling process, i.e. the temperature at which the charge undergoes the carburizing process, and the type and temperature of the cooling agent. This study presents the 
results of comparative analysis of stresses and deformations generated in the charge-bearing, oil- or air-cooled pallets.

\section{Test model}

During heat treatment, both pallet and the charge resting on this pallet are subjected to rapid temperature changes. The process of particularly dynamic character, during which the deformation of the pallet reaches its peak values, is the cooling process. Depending on the heat treatment parameters adopted for the heat treated items, this process occurs with different intensities and in different cooling media. Fluids (oil, water) or gases (e.g. cooling with furnace, in the air outside the furnace chamber, or in neutral gas) are used as coolants [12].

Heat transfer in different cooling agents takes place at different heat transfer coefficients [13, 14], thus producing different intensities of the cooling process. This, in turn, produces different temperatures in the structure of the pallet which has an influence on the stresses and deformations. In this study, temperature changes in a pallet loaded with the charge cooled in two cooling agents, i.e. oil and air, were investigated. Two temperatures of $20^{\circ} \mathrm{C}$ and $150^{\circ} \mathrm{C}$ were adopted for oil cooling.

Figure 1 shows the pallet model adopted in numerical calculations. It represents the casting of a pallet with standard Xtype connections on which the charge in the form of a large concentrated mass is resting. Tests were carried out on pallets with the following dimensions: length $1=620 \mathrm{~mm}$, width $\mathrm{b}=620$ $\mathrm{mm}$, height $\mathrm{h}=50 \mathrm{~mm}$, and wall thickness $\mathrm{d}=8 \mathrm{~mm}$. The charge had the length and width of $500 \mathrm{~mm}$ and the height of $100 \mathrm{~mm}$.

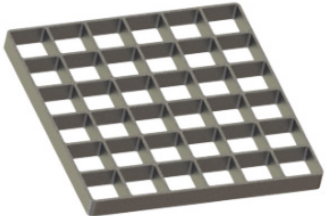

Fig. 1. Pallet model with X-type connections adopted in the studies

Because of the symmetry of the coordinate system in respect of the two major planes, in order to reduce the time needed for complete calculations, the calculations were carried out on $1 / 4$ of the entire model system.

Studies were done in two stages. First, the heat flow was calculated for all the cases examined, and then, based on the obtained temperature distribution, the distribution of stress and deformation was determined. Calculations were done with a Midas NFX 2014 program.

\section{Heat flow analysis}

Three cooling variants from the temperature of $900^{\circ} \mathrm{C}$ were applied in the study, i.e. cooling in air at $20^{\circ} \mathrm{C}$, cooling in oil at $20^{\circ} \mathrm{C}$ and cooling in oil at $150^{\circ} \mathrm{C}$. It has been assumed that heat transfer in the pallet/charge - environment system occurs by free convection, whereas heat flow in the pallet, and between the pallet and the charge placed on this pallet - by conduction.

The same thermal properties of the pallet material and charge material corresponding to the properties of cast steel 1.4849 (GX40NiCrSiNb38-19) according to EN 10295: 2002 [15] were adopted. The specific heat $C_{s}=500 \mathrm{~J} /(\mathrm{kg} \cdot \mathrm{K})$ and the thermal conductivity coefficient $\lambda$ were applied in accordance with the temperature dependence shown in Table 1. The calculations were based on different heat transfer coefficients of the tested cooling agents. For air, a constant value of $h_{s}=0.012 \mathrm{~W} /\left(\mathrm{K} \cdot \mathrm{cm}^{2}\right)$ was adopted. Cooling in oil was based on the function shown in Figure 2 , which allows for the fact that at higher temperatures the coolant should be treated as a boiling and evaporating liquid, the heat transfer coefficient of which differs quite considerably from the coefficient of the same liquid at lower temperatures [11, 13, 14]. The cooling time used in calculations was $400 \mathrm{~s}$.

Table 1.

The coefficient of thermal conduction $\lambda$ of cast steel 1.4849 [15]

\begin{tabular}{ccccc}
\hline Temperature $T,{ }^{\circ} \mathrm{C}$ & 20 & 100 & 800 & 1000 \\
\hline Coefficient $\lambda, \mathrm{W} /(\mathrm{m} \cdot \mathrm{K})$ & 12 & 12.3 & 23.3 & 26.5 \\
\hline
\end{tabular}

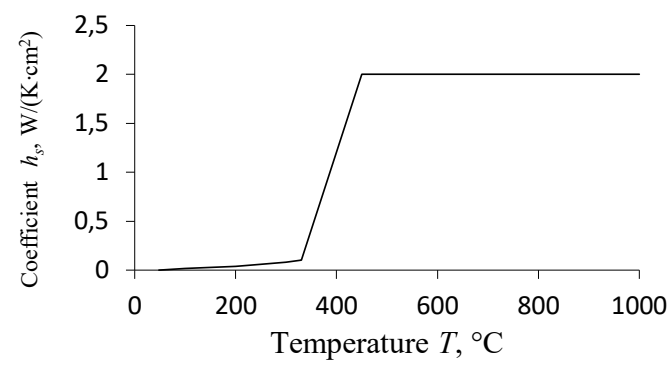

Fig. 2. Heat transfer coefficient of oil

As a result of the calculations, temperature changes were determined in both pallet and charge resting on this pallet. For better illustration, the directions of temperature gradient formation in the pallet structure were marked with five points: $A, B, C, D$ and $E$ (Fig. 3), where points $A, B, C, D$ are lying at half height of the pallet and point $E$ on its upper surface directly under the charge. These points represent the main directions of temperature gradient formation in the pallet structure. The temperature difference between points $A$ and $B$ gives information about the temperature gradient between the core of the pallet wall and its surface at the same height. The temperature difference between points $A$ and $C$ refers to the temperature gradient between the centre of the wall of a regular thickness and the centre of the hot spot formed in the wall connection, whereas the temperature difference between points $A$ and $D$ gives information about the temperature gradient between the fragment of the pallet lying under the charge and its outer contour. The last examined temperature difference between points $A$ and $E$ provides information about the temperature gradient between the point lying in the core of the pallet wall and the point lying directly above and having contact with the handled charge. For all the cases examined, diagrams in Figures 4-7 show the change in temperature differences between the specified points as a function of the cooling time. 

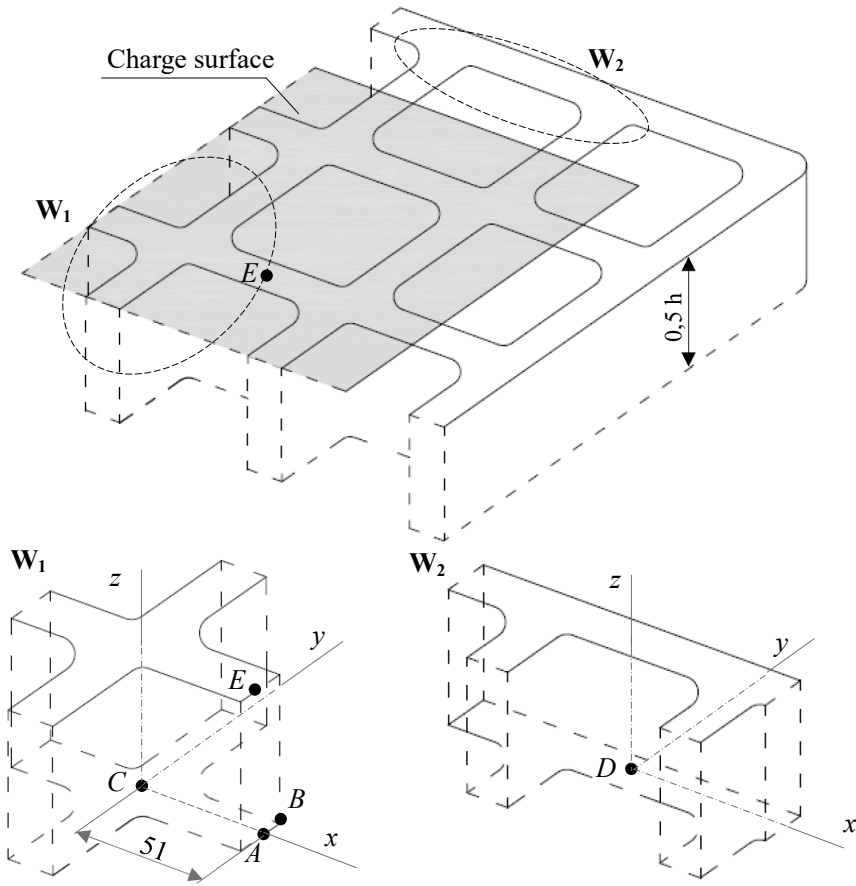

Fig. 3. Temperature measurement points

Respective graphs show changes in the temperature differences between individual points of the pallet as a function of the cooling time. It appears that, in the case of oil cooling, very high temperature differences occur in the first stage of the cooling process between points $A$ and $B$, and $A$ and $C$ (between $A$ and $C$ these differences appear slightly later than between $A$ and $B$ ), and then they either rapidly disappear $(A-B)$ or undergo significant reduction $(A-C)$. In the case of air cooling, practically no major temperature differences exist between points $A$ and $B$, while the difference between points $A$ and $C$ is slowly increasing with the cooling time and its maximum value is much lower than in the case of oil cooling.

The effect of charge is particularly well visible when the temperature difference between point $A$ (lying in the area under the charge) and $D$ (lying in the outer contour of the pallet) is examined (Fig. 6). At the beginning of cooling this difference is very small. However, with the time lapse, when the charge acts as heat accumulator and cooling of the pallet area lying directly underneath is proceeding at a much lower rate, this difference is increasing. From the graph in Figure 6 it follows that the temperature differences between points lying under the charge contour (point $A$ ) and in the outer part of the pallet wall (point $D$ ) increase with the cooling time and reach high values. The calculations show that when air is the cooling agent, the maximum values of these differences are more than three times higher than in the case of oil cooling.

Despite the slower air cooling process, the presence in the pallet structure of so high temperature differences between its internal part under the charge and the outer contour is due to the fact that the pallet itself is a thin wall construction.

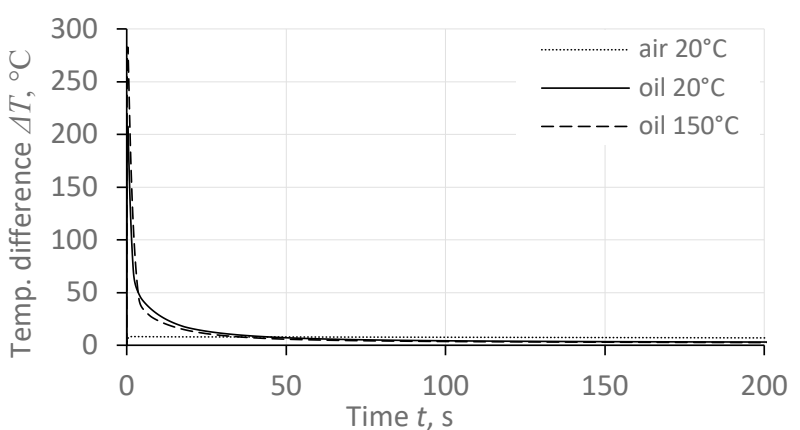

Fig. 4. Change in temperature differences between points $A$ and $B$

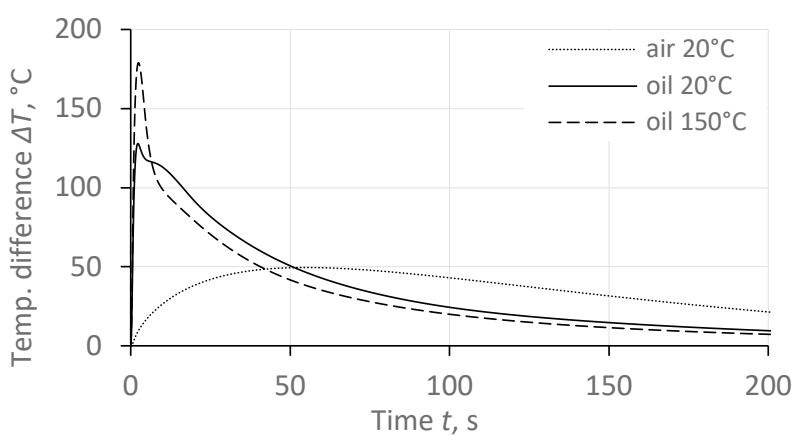

Fig. 5. Change in temperature differences between points $A$ and $C$

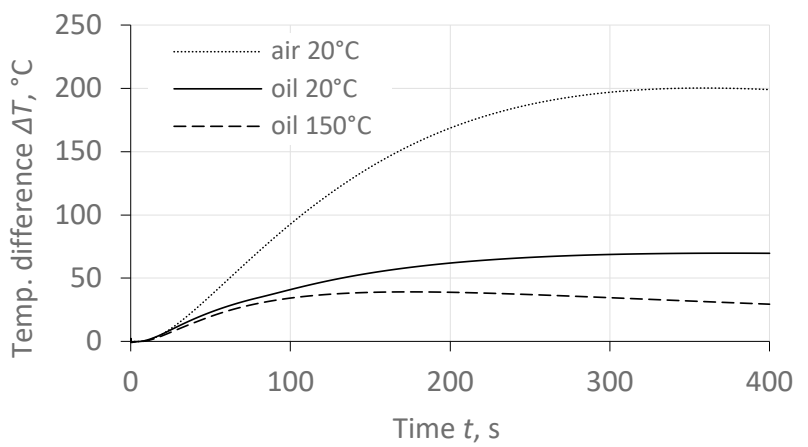

Fig. 6. Change in temperature differences between points $A$ and $D$

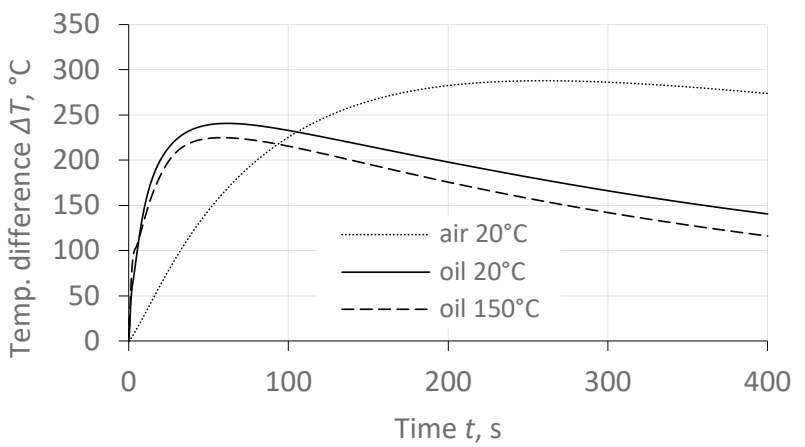

Fig. 7. Change in temperature differences between points $A$ and $E$ 
Although at a rate much lower than in oil, its external walls are cooled significantly faster than the massive charge, for which the temperature remains practically unchanged at first. At the same time, compared to the heat transfer between the inner part of the pallet and the environment, the rate of the heat flow between the charge and the pallet portion lying underneath this charge is high enough to make this difference increase. In the case of oil cooling, due to better heat dissipation from the charge, its outer part is cooled faster than in the air and, at the same time, the rate of heat transfer to the environment in the middle part of the pallet is high enough to consequently make the temperature difference between the inside and outside of the pallet much smaller than in the air (although still significant). This pattern of temperature variations between the inner and outer part of the pallet may result in later stages of cooling in the formation of high stresses and deformations.

The last pair of points where the temperature difference was examined covered points $A$ and $E$ lying on the same vertical axis, i.e. $A$ - in the centre of pallet height and $E$ in the plane of pallet contact with the charge. As in other cases, also now, the effect of charge on the temperature difference between these two points is clearly visible. Careful analysis of the graph in Figure 7 shows the initial significant increase in the temperature difference between points $A$ and $E$, lasting for a considerable period of time (about $60 \mathrm{~s}$ for oil and about $260 \mathrm{~s}$ for air). Then the difference starts decreasing, but due to continuous impact of the charge, this decrease is relatively mild.

\section{Analysis of emerging stresses and deformations}

Based on numerical analysis of the heat flow in pallets cooled together with the charge, simulation analysis of the distribution of thermal stresses and deformations were performed. In calculations, the impact of stresses caused by mechanical loads originating from the charge placed on the pallet has been omitted. As a load in the conducted analysis was adopted the temperature drop in the pallet mesh nodes from the initial value of $900^{\circ} \mathrm{C}$ to the value calculated previously for the time when the temperature difference between points $A$ and $D$ had reached its maximum $T_{(A-D) \max }$ (stage I) and the temperature drop to its final value of $20{ }^{\circ} \mathrm{C}$ or $150{ }^{\circ} \mathrm{C}$ (stage II).

The following mechanical properties of cast steel were used in the calculations: Young's modulus $E=1.73 \cdot 10^{5} \mathrm{~N} / \mathrm{mm}^{2}$, yield strength $R_{e}=208 \mathrm{~N} / \mathrm{mm}^{2}$, the strain-hardening modulus $E_{e}=4.09 \cdot 10^{3} \mathrm{~N} / \mathrm{mm}^{2}$, the thermal expansion coefficient $\alpha=1.77 \cdot 10^{-5}$, Poisson's ratio $v=0.253$ [9].

Figures 8 and 9 graphically depict the results of calculations. Using different shades of grey, the distribution of reduced stresses (calculated according to the Huber-Mises hypothesis) in the pallet structure undergoing cooling in air and oil at $20^{\circ} \mathrm{C}$ is shown, where Figure 8 shows the results for stage I, and Figure 9 - for stage II. In the drawings, darker colours mark areas where stresses have reached a very high value. At the same time, the drawings show the type of deformation caused by an uneven temperature distribution occurring in the charge-loaded pallets during their operating cycle. Given the fact that actual deformations produced in one cycle are very small, they have been represented in multiplied version (same value used in all drawings).
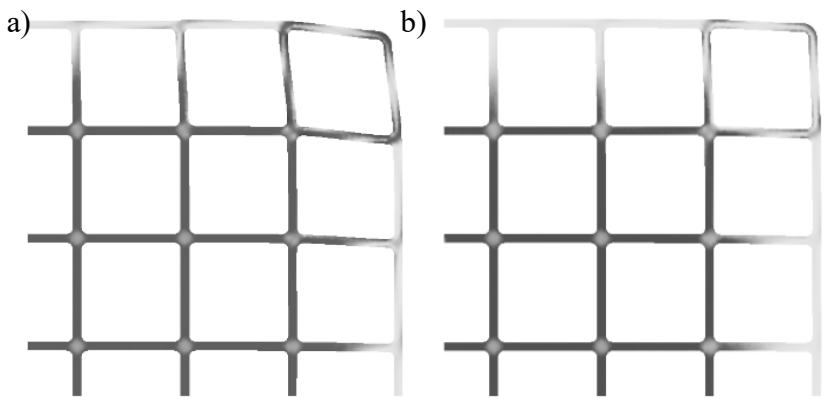

Fig. 8. Deformation and reduced stress distribution in pallet cooled from $900^{\circ} \mathrm{C}$ to $T_{(A-D) \max }$ (stage I): a) in air, b) in oil
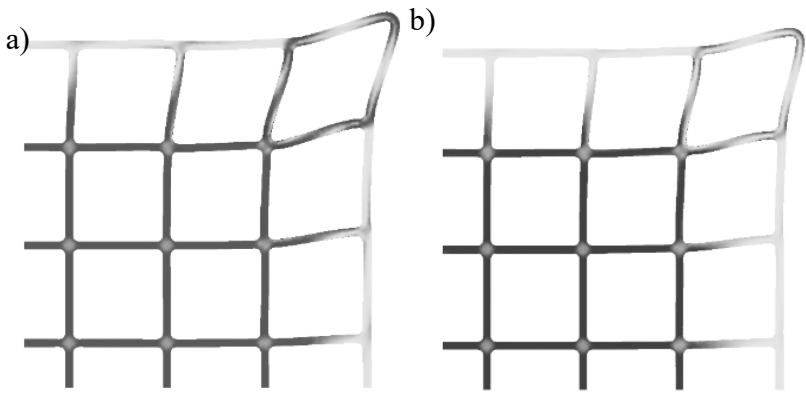

Fig. 9. Deformation and reduced stress distribution in pallet cooled from $T_{(A-D) \max }$ to $20^{\circ} \mathrm{C}$ (stage II): a) in air, b) in oil

The analysis of the results shows that the nature of the resulting deformations is similar irrespective of whether the cooling is carried out in air or oil. In the first stage of this process, the outer contour of the pallet exposed to high rate cooling decreases its dimensions more than other parts of the pallet lying under the charge. This results in the formation of compressive stress in the pallet interior and tensile stress in the pallet walls lying in the outer contour. If at this stage the plastic deformation takes place, then in further part of the cooling process the external contour of the pallet will be squeezed and the internal part stretched. This will cause the characteristic "pushing out" of corners, which is one of the visible symptoms of the deformation occurring in pallets during operation [4].

Comparing the results obtained for two types of coolant, it can be observed that in the case of air cooling the resulting stresses and deformations are greater. This is due to a larger temperature difference between the two pallet zones that are formed during cooling in this type of the cooling agent.

For better comparison, Table 2 shows numerical values of the differences in deformation generated during the first stage of cooling for all the cases considered. This table also shows the displacement in the $y$ direction of points designated in the drawing as $G$ and $F$. Point $G$ lies at the end of a series of walls, most of which are situated under the charge, while point $F$ lies at the end of a series of walls, all of which are situated in the outer contour of the pallet. If temperature changes at various points in the pallet were the same, those points would move by the same distance in the $y$ direction. In practice, however, in the examined fragment of 
the pallet lying under the charge, the decrease of dimensions will be smaller, and consequently, point $G$ will move by a smaller distance than point $F$. The greater is this difference, the larger is the deformation caused by the appearance of temperature gradient. In accordance with the results of the heat flow analysis and stress analysis, the largest difference in displacement between points $G$ and $F$ for stage I will be generated by air cooling. In the case of oil cooling, this difference will be noticeably smaller (slightly higher for oil at $20^{\circ} \mathrm{C}$ than for oil at $150^{\circ} \mathrm{C}$ ).

In Figure 10, in addition to points $G$ and $F$, points $M$ and $N$ are also marked. They both lie in the pallet wall - one point (point $M$ ) in the part of the wall which is under the charge and the other (point $N$ ) in the outer contour of the pallet. Both points lie in the centre of the wall height (Fig. 11) at opposite ends of a straight section of the pallet rib. The distribution of the stress $\sigma_{y}$ and reduced stress $\sigma_{\text {red }}$ in the direction determined by these points is shown in Figures 12 and 13, respectively. It is evident that, like deformation, also high stress is formed in this direction under the conditions of a low air cooling rate. For both cases of oil cooling, these values are similar (again slightly higher for cooling in oil at a lower temperature).

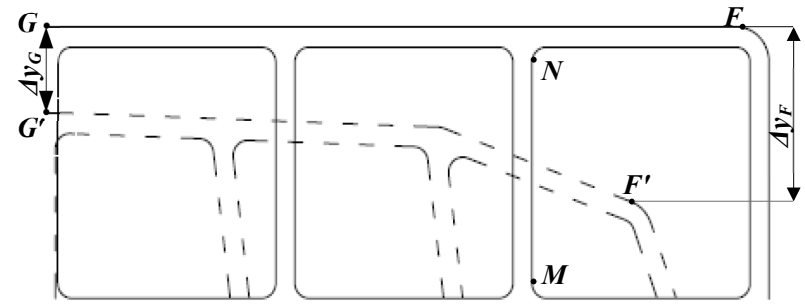

Fig. 10. Displacement of points $F$ and $G$ lying on the pallet contour after the first cooling stage

Table 2

Displacement of points $F$ and $G$ in the $y$ direction after the first cooling stage

\begin{tabular}{cccc}
\hline Coolant & $\Delta y_{G}, \mathrm{~mm}$ & $\Delta y_{F}, \mathrm{~mm}$ & $\Delta y_{F-G}, \mathrm{~mm}$ \\
\hline Oil $20{ }^{\circ} \mathrm{C}$ & 3.75 & 4.4 & 0.65 \\
\hline Oil $150^{\circ} \mathrm{C}$ & 3.43 & 4.00 & 0.57 \\
\hline Air $20^{\circ} \mathrm{C}$ & 2.74 & 4.39 & 1.65 \\
\hline
\end{tabular}

From the analysis of the distribution of stresses $\sigma_{y}$ along the side of the pallet wall it is apparent that, while at one end high tensile stress will be produced, the other end will show the presence of stresses of a similar value but compressive in nature. At the same time, on the opposite side of the pallet wall, stresses of an opposite sign will arise.

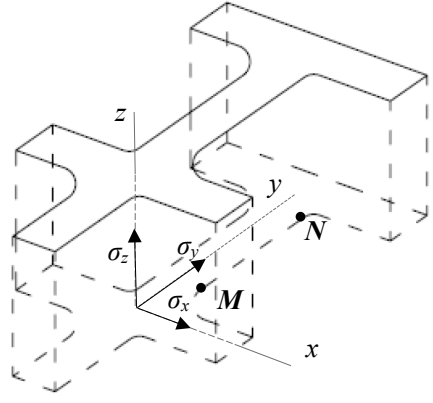

Fig. 11. Components of stress and their directions

With the between the outer and inner fragments of the pallet start decreasing, the situation will reverse, which means that the tensile stresses will replace the compressive stresses, and vice versa. Consequently, in subsequent cycles, the walls of the pallet will be subjected to the effect of bending stresses. The reduced stresses of the highest value will appear at the end of the wall, and precisely at that point, the largest deformation is to be expected.

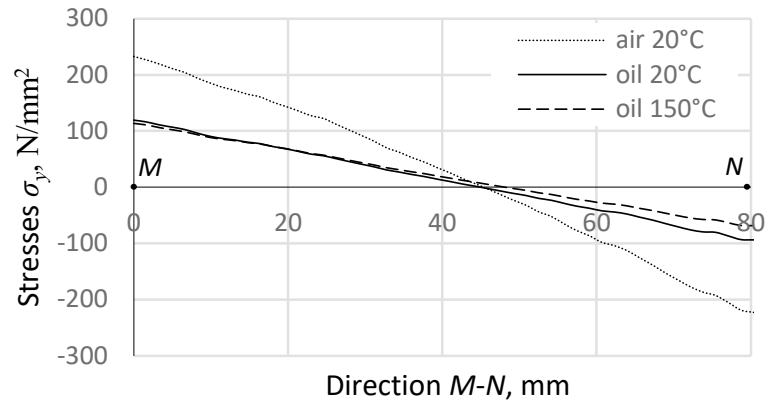

Fig. 12. Distribution of stresses $\sigma_{y}$ in the $M-N$ direction

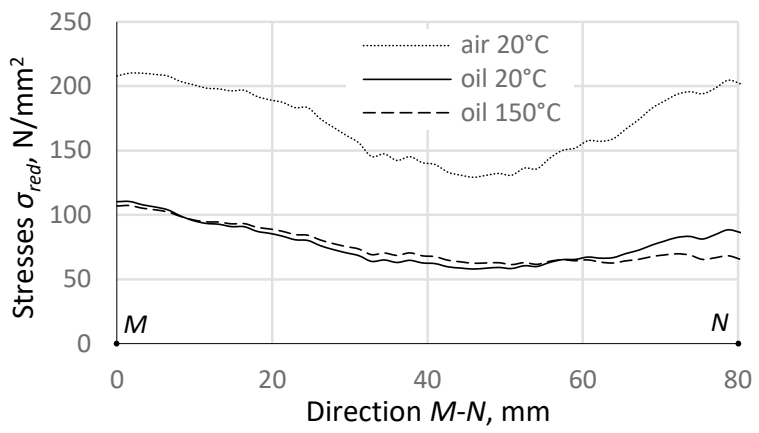

Fig. 13. Distribution of stresses $\sigma_{\text {red }}$ in the $M-N$ direction

From the analysis of the distribution of reduced stresses shown in a pictorial way with different shades of grey in Figures 8 and 9, it follows that high stresses values arise not only in the connections between the outer zone of the pallet and its interior and in the corners of the pallet, but also in the whole area of the pallet lying under the charge. The existence of high stresses in this area is due to high temperature gradient in the $A-E$ direction (Fig. $8)$. The temperature of the pallet material that enters into direct 
contact with the charge (point $E$ ) is much higher than the temperature in the lower layers (point $A$ ), which means that in the first stage the reduction of the dimensions is less drastic. For the examined cases, the stress distribution in the $A-E$ direction is shown in Figure 14. The non-uniform stress distribution in this direction results in the deformation, which takes the form of a protuberance - the middle part of the pallet rises with respect to the outside part (similar behaviour is observed in bimetals subjected to temperature changes). If this deformation is large enough to cause plastic deformation, the situation will reverse in further process of cooling and the outer contour will be lifted in respect of the pallet centre. This deformation is particularly unfavourable when the pallet construction forms the underside part of the basket as it can lead to a relatively quick and visible bending of the side walls.

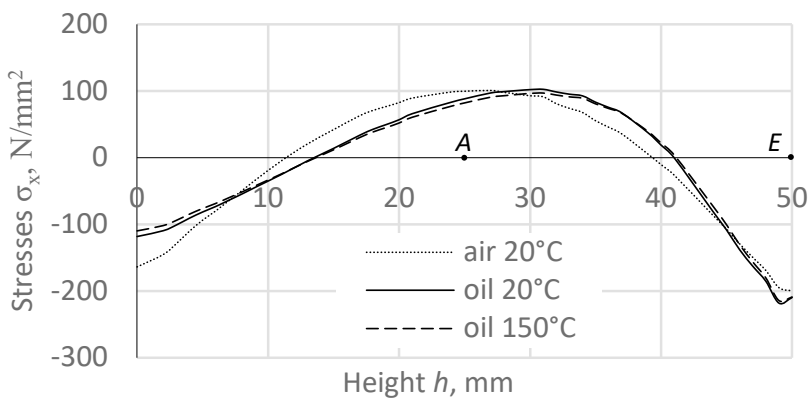

Fig. 14. Distribution of stresses $\sigma_{x}$ in the $A-E$ direction after the first cooling stage

\section{Summary}

The conducted analysis shows that cooling in oil results in the formation of high temperature gradient in the first few moments of process duration in the direction of both transverse $(A-B$ direction) and longitudinal ( $A-C$ direction) wall section near the places where hot spots are present. The resulting temperature differences are much lower when cooling is conducted in air.

The situation is different in the case of temperature differences between the outer zone of the pallet and its interior lying under the charge. Then, when cooling is carried out in air, these differences reach much higher values, which results in higher levels of both stress and deformation. Stresses of this type are particularly dangerous for the pallet construction because they expose the walls to the damaging effect of cyclically varying tensile and compressive loads. The additional consequence is "pushing out" of the pallet corners which, with a sufficiently large number of the operating cycles and growing deformation, can also result in decommissioning of the pallet.

Temperature differences examined in the vertical direction $(A-$ $E$ ) have yielded the results similar for different cooling methods and comprised basically in the same range of values.

The information regarding the effect of the cooling agent on the cooling kinetics and related stresses and deformations is important for designers of the heat treatment furnace accessories. It is true that they have practically no effect on the choice of the cooling agent in which parts of the furnace accessories used for handling of the heat treated charge operate, but at least they know what kind of cooling agent will be applied. Knowing how individual cooling agents affect the temperature gradients during subsequent heat treatment steps, they can propose some structural modifications to minimize the most dangerous (under given cooling conditions) stresses.

Further activities in this field should focus on studies of the impact of charge weight and distribution on the resulting stresses, and on the methods of quantitative assessment of the contribution of various technological solutions to the optimization of pallet structure considered in terms of the arising thermal stresses.

\section{References}

[1] Lai, G.Y. (2007). High-Temperature Corrosion and Materials Applications. ASM International.

[2] Davis, J.R. (Ed.). (1997). Industrial Applications of HeatResistant Materials. In Heat Resistant Materials (67-85). ASM International.

[3] Dossett, J.L., Boyer, H.E. (2006). Practical Heat Treating. ASM International, 2nd ed.

[4] Piekarski, B. (2012). Creep-resistant castings used in heat treatment furnaces. Szczecin: West Pomeranian University of Technology Publishing House. (in Polish).

[5] Piekarski, B. \& Drotlew, A. (2008). Designing of castings working in conditions of temperature cyclic changes. Archives of Mechanical Technology and Materials. 28(3), 95-102. (in Polish).

[6] Drotlew, A. et al. (2013). Structure of guide grate in heat treatment technological equipment. Transactions of foundry research institute. 53(3), 59-71, DOI: 10.7356/iod.2013.16.

[7] Słowik, J.A, Drotlew, A. \& Piekarski, B. (2016). The flexibility of pusher furnace grate. Archive of Foundry Engineering. 16(4), 137-140. DOI: 10.1515/afe-2016-0098.

[8] Lo, K.H. et al. (2009). Recent developments in stainless steels. Materials Science and Engineering R. 65, 39-104.

[9] Gutowski, P. (1989). Research the causes of cracking in pallets operating in furnaces for the carburising treatment. Unpublished doctoral dissertation, Technical University of Szczecin, Szczecin. (in Polish).

[10] Bajwoluk, A. \& Gutowski, P. (2015). The effect of pallet component geometry on temperature gradient during cooling. Archive of Foundry Engineering. 15(1), 5-8. DOI: 10.1515/afe-2015-0001.

[11] Bajwoluk, A. \& Gutowski, P. (2014). Analysis of thermal stresses in components of pallets operating in furnaces for the carburising treatment. Archive of Foundry Engineering. 14(spec.1), 175-180.

[12] Luty, W. (1986). Quenching cooling agents. Warszawa: Science-Technical Publishers. (in Polish).

[13] Orłoś, Z. (1992). Thermal stresses. Warszawa: Polish Scientific Publishers. (in Polish).

[14] Petela, R. (1983). Heat flow. Warszawa: Polish Scientific Publishers. (in Polish).

[15] Standard EN 10295:2002. Heat resistant steel castings. 